\section{Cahiers de Narratologie}

Analyse et théorie narratives

$13 \mid 2006$

Nouvelles approches de l'intertextualité

\title{
Intertextualité et humour dans Le taureau blanc de Voltaire
}

Marie-Hélène Cotoni

\section{CpenEdition}

Journals

Édition électronique

URL : http://journals.openedition.org/narratologie/323

DOI : $10.4000 /$ narratologie.323

ISSN : 1765-307X

Éditeur

LIRCES

Référence électronique

Marie-Hélène Cotoni, « Intertextualité et humour dans Le taureau blanc de Voltaire », Cahiers de Narratologie [En ligne], 13 | 2006, mis en ligne le 01 septembre 2006, consulté le 19 avril 2019. URL http://journals.openedition.org/narratologie/323 ; DOI : 10.4000/narratologie.323

Ce document a été généré automatiquement le 19 avril 2019

\section{(c) $($ i) $(9)$}

Cahiers de Narratologie - Analyse et théorie narratives est mis à disposition selon les termes de la licence Creative Commons Attribution - Pas d'Utilisation Commerciale - Pas de Modification 4.0 International. 


\title{
Intertextualité et humour dans Le taureau blanc de Voltaire
}

\author{
Marie-Hélène Cotoni
}

1 Si intertextualité et humour impliquent, l'un et l'autre, hétérogénéité discursive ou discontinuité, ils ont aussi en commun de recevoir, selon les critiques, des acceptions d'extension variable... Mon approche de l'intertextualité sera moins restrictive que celle de G. Genette, puisque je vais inclure ici ce qu'il analyse, dans Palimpsestes, sous le nom d'hypertextualité. Et dans les relations, de coprésence ou de dérivation, entre un conte voltairien et l'Ancien Testament, je tenterai de cerner avec précision ce qui relève de l'humour. C'est-à-dire d'un "esprit de la pensée $»^{1}$, d'un jeu peu agressif, où des associations étranges, que le narrateur feint de nous présenter avec le même sérieux que s'il n'en voyait pas l'incongruité, aboutissent à une représentation surréelle.

2 Un des livres le plus souvent lus et commentés par Voltaire est indiscutablement la Bible. Il a laissé des marques de lecture, en quantité inégale, il est vrai, dans chacun des vingtquatre volumes du Commentaire littéral sur tous les livres de l'Ancien et du Nouveau Testament, du bénédictin dom Calmet ${ }^{2}$. Dans ses œuvres publiées, comme dans sa correspondance, les références à la Bible sont fréquentes, beaucoup plus que chez Montesquieu, Rousseau ou Diderot. On sait avec quelle véhémence, à travers ses "examens", "sermons", « homélies », « catéchismes », sans parler du Dictionnaire philosophique, ou de La Bible enfin expliquée, écrite deux ans avant sa mort, il a exprimé sa répugnance à l'égard de l'irrationalité et de l'immoralité qu'il voyait dans nombre de textes bibliques. Il résume alors l'Ecriture à sa manière, puis juxtapose interrogations, difficultés, hypothèses, commentaires, afin de dénoncer l'imposture qui fait du moindre verset, selon l'Eglise de son temps, la parole même de Dieu, ainsi que le fanatisme et le despotisme du clergé, qui découlent de cette certitude. Le Livre est donc l'objet, de la part du philosophe, d'un regard critique et d'un discours second, qui tend d'ailleurs, par les professions de foi déistes qui s'y ajoutent, à se substituer à la Révélation initiale.

3 Mais le poids de ces polémiques ne doit pas nous faire négliger une autre approche de l'Ecriture, visible dans sa correspondance, et dans quelques-uns de ses récits. Quand il n'est pas horrifié par les innombrables massacres relatés dans l'Ancien Testament, et 
surtout par les atrocités modernes qu'on justifie en s'y référant, Voltaire aime lire la Bible. En septembre et octobre 1759, il en conseille la lecture à Madame du Deffand, pour " la singularité des mœurs antiques », « la foule des événements dont le moindre tient du prodige », la « naïveté ». Il tente de la persuader que chaque page fournit « des réflexions pour un jour entier ", que ce livre " fait cent fois mieux connaître qu'Homère les mœurs de l'ancienne Asie. $»^{3}$. Comme l'écrit René Pomeau, en même temps la Bible «le dégoûte et (...) l'enchante $»^{4}$.

Cette réaction, chez un lecteur qui se régale aussi des Mille et une nuits, explique que, devant les versets de l'Ecriture, la dimension ludique puisse parfois s'ajouter ou se substituer à la dimension polémique. C'est particulièrement fréquent dans la correspondance ${ }^{5}$, les destinataires étant assez imprégnés de culture biblique pour qu'une connivence s'établisse. C'est également le cas dans un conte tardif, publié en 1774, où domine le merveilleux et où l'écrivain, à quatre-vingts ans, retrouve une vivacité désinvolte pour broder sur des fables, le seul conte de critique biblique du XVIII ${ }^{e}$ siècle selon René Pomeau ${ }^{6}$, Le taureau blanc.

Cette fantaisie orientale mêle tous les ingrédients d'un conte de fées : amours contrariées d'une jeune princesse, métamorphose d'un prince en bête, animaux qui parlent. Mais ce sont des réminiscences de la Bible qui inspirent le plus souvent Voltaire. C'est, d'abord, la malédiction prononcée à la fin du chapitre IV du Livre de Daniel ${ }^{7}$, citée partiellement au chapitre $\mathrm{V}$ du conte. Une voix venue du ciel a dit à Nabuchodonosor: « La royauté s'est retirée de toi, /d'entre les hommes tu seras chassé, /avec les bêtes des champs sera ta demeure, / $d$ 'herbe, comme les bœufs, tu te nourriras ». En présentant, comme le faisaient aussi certains apologistes ${ }^{8}$, ce prince littéralement métamorphosé en un taureau «blanc, fait au tour, potelé, léger même ", doté de belles cornes d'ivoire', et en le comparant aux taureaux légendaires qui avaient séduit Pasiphaé et Europe, l'écrivain réduit l'Ancien Testament à un recueil de fables, assimilables à celles de l'Antiquité païenne. Mais, une fois ces équivalences suggérées, même s'il les répète souvent pour mieux convaincre ${ }^{10}$, il va aussi utiliser, selon sa fantaisie, la Bible comme un réservoir d'épisodes merveilleux. Le lecteur doit donc faire appel à sa mémoire, s'il veut goûter toutes les allusions intertextuelles. Car à ce taureau étonnant le narrateur adjoint une gardienne tirée du premier livre de Samuel (XXVIII 7-25), puisqu'il s'agit de la pythonisse d'Endor ${ }^{11}$ qui, à la demande du roi Saül, avait fait apparaître l'ombre de Samuel. Il l'accompagne d'un bestiaire venu de la Genèse (III 1-16 et VIII 6-12), des Nombres (XXII 22-35), de Jonas (II), de Tobie (VI 2) et du Lévitique (XVI 8-10). Sont en effet réunis autour de la vieille, et conversent ensemble, le serpent qui tenta Eve, le corbeau et la colombe qui, les premiers, quittèrent l'arche de Noé, l'ânesse de Balaam, le poisson qui avala Jonas, le chien de Tobie, et enfin le bouc émissaire.

6 Remarquons d'abord que, dès qu'ils ne sont plus articles de foi ${ }^{12}$, l'écrivain se laisse prendre au charme naïf de récits où le surnaturel est mêlé aux détails de la vie rustique. Ici une pauvre ânesse, battue à trois reprises parce qu'elle refusait d'aller où voulait son maître, le prophète Balaam, reproche au brutal ses mauvais traitements: elle n'avait pourtant eu d'autre tort que de voir avant lui un ange qui leur barrait le chemin. Là le fils de Tobie entreprend un voyage lointain : "L'enfant partit avec l'ange, et le chien suivit derrière ». Quant au poisson qui, depuis trois jours, gardait le prophète Jonas dans ses entrailles, il suffit d'un ordre de Yahvé pour qu'il le recrache sur le rivage.

7 A partir de là, Voltaire pratique une surenchère dans la féérie. On va de surprise en surprise, la première étant la révélation progressive de l'identité véritable du taureau, 
puisque s'échappent une à une, de la bouche de la princesse, entre le premier et le neuvième chapitre, les syllabes qui composent le nom de Nabuchodonosor. Le dernier coup de théâtre sera la métamorphose du bœuf en dieu, avant le retour à la forme humaine. Certes, en rivalisant d'imagination avec les auteurs anciens, l'écrivain veut montrer que la Bible n'est qu'un conte. Mais cette volonté de désacralisation n'ôte rien au charme de l'histoire qu'il narre. L'imbrication de deux écritures - l'invention voltairienne s'exerçant sur une base biblique - produit de constants décalages qui peuvent être source d'humour. En effet, si l'humour, selon la définition de Jean Emelina, est «paradoxe tranquille $\aleph^{13}$, il innerve tous les épisodes où le narrateur, avec la plus grande impassibilité, fait coexister les traits bibliques de ses personnages avec des comportements étonnants et des situations inattendues. Il naît également de la pratique insouciante de dérapages chronologiques, de paralogismes, de passages abrupts d'un genre littéraire à un autre, de disparates stylistiques, et du jeu entre le dit et le non dit.

8 Toutefois, en ce qui concerne les personnages humains, quand Voltaire juxtapose deux " vérités ", il décèle la médiocre réalité derrière la légende. L'intention satirique est alors trop évidente pour laisser beaucoup de place au jeu gratuit. Dès le début du conte, on passe du noble au burlesque, avec un apparent naturel. Le conseiller de la princesse Amaside, « le sage Mambrès, ci-devant sorcier de pharaon » (362), âgé de treize cents ans, est celui qui, selon l'Exode, rivalisa avec Moïse. L'écrivain est allé chercher son nom dans la deuxième épître de Paul à Timothée (III 8). Or ce personnage hors du commun, qu'il honore d'un beau décasyllabe, «il fallut des dieux pour vaincre Mambrès » (359), ce « divin Mambrès » admet que, tout prophète qu'il est, il sait «bien peu de choses » (375). Et c'est par la seule habileté de son esprit qu'il parviendra à réunir la princesse et son bien-aimé, en sauvant ce dernier du courroux du roi Amasis.

Le travestissement burlesque est plus appuyé quand apparait la pythonisse d'Endor. Le narrateur juxtapose ironiquement les nobles titres que lui donne Mambrès, « respectable dame », « auguste pythonisse » (362), «illustre pythonisse » (364) et la représentation réaliste d'une sorcière, «une vieille femme couverte de lambeaux gris » (360). Cette démythification justifie qu'elle soit désignée par les termes de «la vieille » (passim), « ma bonne » ou, en un plaisant anachronisme, « mademoiselle d'Endor » (364), d'où Amaside conclura qu'elle est pucelle!

10 Aussi la réminiscence de deux épisodes étranges de l'hypotexte (I Samuel XXVIII 11-19 et Daniel II) n'aboutit qu'à l'invention d'une péripétie décevante : la sorcière a beau faire apparaitre, toutes les nuits, des ombres au roi Amasis, pour le détourner de ses idées de meurtre, elle ne parvient à rien, puisqu'au matin il a oublié ses songes. Voilà mis en échec le surnaturel!

11 La vision que le lecteur a des trois prophètes qui débarquent, à la fin du chapitre $\mathrm{V}$, est construite sur les mêmes décalages : air grave, majestueux, auguste, figuré par les traits de feu qui partent de leur tête, contrastant avec les «lambeaux crasseux et déchirés » dont ils sont couverts (379). La coexistence tranquille des attributs traditionnels et de la dérivation burlesque pourrait laisser filtrer l'humour. Mais il est brouillé par l'intention satirique.

En revanche, le dérèglement ludique l'emporte quand le conteur nous détaille avec complaisance le repas succulent ${ }^{14}$ servi aux trois prophètes Daniel, Ezéchiel et Jérémie. D'une part, l'anachronisme d'un luxueux menu du XVIII ${ }^{e}$ siècle, d'autre part les allusions intertextuelles conduisent à une logique incongrue. En effet, Daniel a failli servir lui même de repas quand il était dans la fosse aux lions (Daniel VI), malgré son affirmation 
sentencieuse et invérifiable: «Monsieur, vous savez que les lions ne mangent jamais de prophètes » (381). Jérémie a, de son propre aveu, passé toute sa vie à mourir de faim. Quant à Ezéchiel, on sait combien Voltaire s'est plu à mentionner, à partir d'une interprétation discutable (Ezéchiel IV 9-15), les fameuses tartines d'excréments que Dieu lui aurait ordonné de manger. Le narrateur ne manque pas, ici, d'y revenir ! Le discours nous ramène alors à la satire.

Passons sur la métamorphose des prophètes en pies. Elle dérive encore doublement de leur passé biblique puisqu'ils se sont toujours montrés de grands parleurs et qu'ils ont fait œuvres pies. Mais le comique, moins délicat, tient à l'esprit des mots, à l'énoncé et non à l'énonciation.

C'est surtout avec les animaux bibliques que, tout en sous-entendant partout, on l'a vu, une critique du fabuleux, le conteur joue le jeu. Il laisse au taureau et au serpent la double nature qu'ils tiennent de l'hypotexte. Il peut donc feindre de vouloir nous persuader que, dans les yeux bovins de l'ancien amoureux de la princesse, règne, quand il la regarde, « un mélange inouï de douleur et de joie » (361), que ce taureau sait pleurer, se jeter aux pieds de celle qu'il aime et les baiser, ou, l'air soumis, plier les quatre genoux. Amaside pourra venir le caresser, lui donner des biscuits et le faire danser. Traits anthropomorphiques que l'ambivalence du personnage, d'après la Bible, peut expliquer, mais qui n'en font pas moins sourire. Comme le récepteur sourira de l'alliance inverse, faite le plus sérieusement du monde, entre deux éléments incompatibles: un agent réintroduit dans une catégorie humaine privilégiée et une action propre à l'animalité : "Ce grand et malheureux prince broutait l'herbe auprès de la tente " (381). Dans ce cas, selon la formule de D. Noguez, "le signifié lui-même, par son 'énormité', signifie sa propre nullité $»^{15}$.

Le passé mythique du serpent tentateur justifie également certains qualifiants étonnants, ou ambigus, tranquillement donnés pour naturels. C'est " un beau serpent ", peut-être « une personne de la plus grande considération », qui «a de l'esprit» et «s'explique en bons termes ", qui "est fort poli avec les dames", mais est "pétri d'amour-propre " (365). Paradoxalement ses yeux de reptile sont « aussi tendres qu'animés » (360). En guise de réplique au discours qu'il avait tenu à Eve, le conteur nous gratifie d'une conversation galante entre lui et la princesse, lorsqu'elle a obtenu, selon une formule cocasse, " un tête-à-tête avec le serpent » (366). Comme, aux yeux du philosophe, goûter le fruit de l'arbre qui donnait la science du bien et du mal ne saurait être un danger, euphémismes et expressions familières réduisent à une plaisanterie sans conséquence la tragique histoire de la chute : le serpent s'est borné, autrefois, à jouer « un tour pendable aux hommes » (367).

Le conte, en effet, exclut le tragique et le symbolique. Face au barbare Amasis et à ses alliés, tous les personnages, comme l'indiquent les possessifs, font partie d'un petit monde familier, intime, solidaire : Mambrès va trouver « sa pythonisse ", pour lui exposer « la fantaisie de sa princesse » (366). La pythonisse décide de conduire son taureau « loin des cruautés du roi de Tanis ", ajoutant : «Mon poisson et mon serpent me défendront » (376). L'ombre de La Fontaine plane sur ces bêtes proches de l'homme.

17 Même le souvenir de la fille de Jephté, dont Voltaire s'est si souvent servi pour affirmer l'existence, chez les Juifs, de sacrifices humains ${ }^{16}$, aboutit à un décrochage humoristique. Car dans l'univers feérique du conte, les événements les plus graves ne sont jamais pris au sérieux. L'impassibilité du conteur dans certains titres de chapitres, le dixième par exemple, «Comment on voulut couper le cou à la princesse, et comment on ne le lui 
coupa point » (388), montre bien l'innocuité réelle des annonces les plus redoutables. Aussi, quand Amaside, condamnée à mort par son père, lui demande, comme la fille de Jephté, dans une parodie de l'épisode biblique, un peu de temps pour pleurer sa virginité, la réponse s'agrémente d'une chute comiquement grivoise: "Je vous laisse toute la journée pour pleurer votre virginité, puisque vous dites que vous l'avez » (388).

La désacralisation entraine également la suppression de tout symbolisme, au profit de l'absurde ${ }^{17}$, puisque n'est conservée qu'une gestuelle dépourvue de signification. Lorsque Mambrès-Voltaire décide d'envoyer le bouc émissaire dans le désert, il ajoute, avec toute l'apparence du sérieux : «L'on sait que tout s'expie avec un bouc qui se promène » (375).

Les autres animaux venus de la Bible, chien, ânesse, colombe, poisson semblent agir selon la fantaisie la plus arbitraire. En dehors de la mission dont Mambrès charge les trois premiers, en raison de leurs antécédents, le narrateur s'amuse à donner une autonomie nouvelle à ces " êtres de papier ", à leur prêter même une gesticulation insolite. Lorsque la princesse Amaside reconnaît son cher Nabuchodonosor dans le taureau blanc et s'évanouit,

le serpent est attendri : il ne peut pleurer, mais il siffle d'un ton lugubre ; il crie : « Elle est morte ! » L'ânesse répète : « Elle est morte ! » Le corbeau le redit ; tous les autres animaux paraissent saisis de douleur, excepté le poisson de Jonas, qui a toujours été impitoyable ( 371).

21 Au comique de situation, à la mécanisation née des répétitions s'ajoute une trouvaille inattendue: le trait particulier prêté au poisson, sans raison aucune. La scène atteint l'extravagance.

Toutefois le corbeau est à peu près exclu de cet univers ludique. C'est probablement à la couleur de son plumage qu'il doit le mauvais rôle qui lui est attribué. Rappelons-nous, dans Candide, le "petit homme noir, familier de l'Inquisition» et dans La princesse de Babylone, « la nation vêtue de noir ", au-delà des Pyrénées, en pays d'Inquisition ${ }^{18}$. Aussi le vilain corbeau remplit-il la fonction de délateur auprès du roi Amasis. C'est à lui, également, que Voltaire prête un long discours dont les invraisemblances sont dignes du plus sot des apologistes. L'intention ironique l'emporte alors. En effet, un des arguments utilisés pour contester la réalité du déluge universel était l'impossibilité d'avoir fait tenir dans l'arche et nourri pendant de longs mois des animaux de toutes les espèces :

Nous y faisions très bonne chère, repartit le corbeau. On servait du rôti deux fois par jour à toutes les volatiles de mon espèce.(...) Il y avait dans l'arche huit personnes de marque et les seules qui fussent alors au monde, continuellement occupées du soin de notre table (...) (378).

Toutefois, même au milieu de traits satiriques appuyés, lorsqu'au chapitre VIII, le serpent, porte-parole de Voltaire, entreprend de ruiner l'autorité de la Bible, il arrive que la critique se fasse humoristique, en dévoilant l'absurde par l'énoncé tranquille d'un paralogisme: après avoir fait pleuvoir de grosses pierres sur un bataillon d'ennemis, Josué « les ayant ainsi exterminés, arrêta le soleil et la lune en plein midi, pour les exterminer encore » (384).

Mais c'est surtout lorsque le conteur cesse de polémiquer que l'humour reprend ses droits. Nous avions relevé quelques anachronismes. Tout au long du texte, ils ajoutent des incohérences qui contribuent encore davantage à la «mise en vacances du principe de réalité $»^{19}$. Déjà la présence simultanée de Mambrès, contemporain de Moïse, de la pythonisse, contemporaine de Saül, de Nabuchodonosor et enfin des animaux empruntés 
à des livres, donc à des siècles différents, repose sur un véritable brouillage chronologique ${ }^{20}$, dont l'auteur est évidemment conscient, même s'il n'en a pas l'air. Que dire, alors, quand le serpent annonce Le paradis perdu de Milton, quand la princesse mentionne Abbadie et Houtteville, puis une œuvre de Locke, un conte de La Fontaine, et enfin Rollin, quand il est dit qu'Amphion bâtissait une ville au son du violon, le temps de danser un menuet ou un rigodon, quand Mambrès écrit «sur du papier d'Egypte qui n'était pas encore en usage » (376), ou quand les prophètes prennent le café avant de se promener au bord du Nil ?

A toutes ces extravagances s'ajoute une volontaire confusion des genres, lorsque des fictions sont présentées avec une précision et des références dignes d'un ouvrage historique, que les Métamorphoses d'Ovide sont qualifiées d'« histoire véritable » (374) et que, malicieusement, l'auteur joue sur la relation entre son conte et d'autres fables, au moment où il évoque la transformation en pies des filles de Pieros.

De même, les disparates stylistiques désorientent. Est-on dans l'univers de l'épopée ? On le croirait à lire les belles comparaisons magnifiant notre taureau. Est-on dans l'univers de la tragédie ? On le pense quand on entend les lamentations d'Amaside, mêlées de vers blancs : « $O$ mon amant ! mon jeune et cher amant! (...) Quel dieu t'a enlevé à ta tendre Amaside? " (360) Ou quand on assiste à la scène de reconnaissance entre le mage et la pythonisse, à laquelle ne manquent ni les exclamations, ni l'invocation de la Providence. Les entretiens entre la princesse et le taureau empruntent au style de l'idylle. On retrouve, d'autre part, la simplicité biblique quand l'ânesse répond à Mambrès : « Comme j'ai servi Balaam, je servirai monseigneur ; j'irai et je reviendrai » (377). Pastiche, parodie, dérapages vers le burlesque se succèdent, et cette succession sereinement anormale touche à l'humour. Cette virtuosité est signe de liberté, signe que nous sommes dans l'univers du conte, où tout est jeu, où règne l'arbitraire. Le conteur favorise l'humour quand il fait sa part à l'altérité. L'amalgame générique et stylistique correspond également, par rapport à l'expression littéraire classique, à une surréalité.

Enfin, plus que les jeux sur les mots « chérubin », « impayable » (362) ou «bœuf» (391), qui tiennent à l'énoncé et non à l'énonciation, les sous-entendus érotiques, les euphémismes, les vacillements de sens relèvent aussi de l'humour. Quelles sont les intentions du taureau quand il invite la princesse à venir le "voir quelquefois sur l'herbe »? (364) Et à quoi pense le conteur quand il qualifie Loth de "plus tendre père qu'on ait jamais vu »? (374) 21

28 Certes, Haydn Mason l'a montré22, les traits satiriques visant la Bible ne manquent pas: emprunts des Juifs aux autres religions, propagation très limitée des fables juives, grossièretés et obscurité de certains textes prophétiques, invraisemblances, immoralités. Pas plus qu'ailleurs Voltaire n'épargne, dans ce conte, Elie, Josué, Samson, Oolla ou Ooliba. N'oublions pas, non plus, que lorsqu'il raconte avec le plus grand sérieux une histoire incroyable, il invite au scepticisme à l'égard de tous les récits du même genre.

Mais ne pas croire à ces histoires n'interdit pas d'y trouver du plaisir. La saveur particulière de ce conte vient de l'entrelacement serein de fables nouvelles et de fables anciennes, de la création, avec les animaux surtout, d'un univers au-delà de la réalité, étrange, extravagant parfois, d'un «ballet malicieux et poétique ${ }^{23}$. Il s'agit ici d'un humour léger, correspondant à ce que Morier nomme une «ironie de conciliation " ${ }^{24}$, puisque le conteur s'amuse à broder sur le canevas d'un imaginaire ancien pour nous offrir, avec un sérieux impassible, des aventures cocasses. Des aventures qui s'achèvent sur le meilleur des dénouements possibles, dans une même sympathie pour les hommes 
et pour les bêtes. Si, selon la formule de V.Jankélévitch, «humoriser c'est ironiser en regardant au loin et au-delà $»^{25}$, Voltaire humorise encore dans Le taureau blanc quand, après ses railleries acerbes sur la Bible, il redit, par un détail futile du dénouement, la distance entre la réalité et la fable : le bouc émissaire, après avoir passé un jour dans le désert, reçoit "douze chèvres pour sa récompense » (391). Mais par sa fantaisie toute gratuite, cette trouvaille exprime aussi le charme du merveilleux, et d'un conte où la virtuosité le dispute à une naïveté parfaitement simulée.

\section{NOTES}

1. Freud, Le mot d'esprit, Paris, Gallimard, 1978, p. 145.

2. Voir le Corpus des notes marginales de Voltaire, tome II, Berlin, Akademie-Verlag, 1982, p. 22-323.

3. Correspondence and related documents, éd. Th. Besterman, Oxford, 1968-1977, D 8484 et 8533. Pour des détails complémentaires, voir notre chapitre «Voltaire lit la Bible» in Voltaire en son temps, sous la direction de R. Pomeau, Oxford et Paris, Fayard, 1995, tome II, p. 178-189.

4. Introduction au Taureau blanc, Paris, Nizet, 1956, p. XXXIX.

5. Voir notre article : " Présence de la Bible dans la correspondance de Voltaire ", Studies on Voltaire and the eighteenth century, 319, 1994, p. 357-398, et la thèse de François Bessire, La Bible dans la correspondance de Voltaire, Studies on Voltaire, 367, Oxford, 1999.

6. Introduction citée, p. XXXV.

7. Marqué par un signet dans l'exemplaire du Commentaire de dom Calmet conservé dans la bibliothèque de Voltaire (Corpus... II, p. 228).

8. A commencer par dom Calmet, dans sa Dissertation sur la métamorphose de Nabuchodonosor, tandis que d'autres préfèrent l'hypothèse de la lycanthropie.

9. Contes en vers et en prose, éd. Sylvain Menant, Classiques Garnier, Paris, Bordas, 1993, tome II, p. 361. Les références entre parenthèses, dans le texte, renverront à cette édition. 10. Le thème de la métamorphose revient souvent dans le conte, avec la mention d'autres épisodes bibliques et des références aux Métamorphoses d'Ovide, comme dans l'article « Métamorphose » du Dictionnaire philosophique, ou le chapitre 29 de La Philosophie de l'histoire : « Les métamorphoses recueillies par Ovide (...) ne devaient point du tout étonner un pythagoricien, un brame, un Chaldéen, un Egyptien (...) Les Juifs dans des temps très postérieurs écrivent que Nabucodonosor fut changé en boeuf, sans compter la femme de Loth transformée en statue de sel. » (O.C. t. 59, p. 186).

11. Il l'avait déjà introduite, chevauchant un balai, dans La Pucelle (chant IV) et dans Saül (Acte II).

12. Il est inutile de rappeler les nombreux traits satiriques lancés contre des puérilités incompatibles, aux yeux de Voltaire, avec la parole de Dieu. Nous ne citerons qu'un exemple, tiré du Catéchisme de l'honnête homme : Après avoir indiqué quelle religion l'Etre Suprême a gravée dans tous les cœurs, adoration de Dieu et amour du prochain, le philosophe ajoute : « Mais certes il n'y a pas gravé que trois font un, qu'un morceau de 
pain est l'Eternel, et que l'ânesse de Balaam a parlé. » (Mélanges, Bibliothèque de la Pléiade, 1961, p. 669).

13. Le comique, Paris, SEDES, 1991, p. 145.

14. Sur les cinq services de ce dîner, voir Christiane Mervaud, Voltaire à table, Paris, éd.

Desjonquères, 1998, p. 69.

15. «Structure du langage humoristique », Revue d'esthétique, t. 22, p. 45.

16. Voir, dans le Dictionnaire philosophique, l'article «Jephté, Ou des sacrifices de sang humain » : « Il est évident par le texte du livre des Juges que Jephté promit de sacrifier la première personne qui sortirait de sa maison pour venir le féliciter de sa victoire contre les Ammonites. Sa fille unique vint au-devant de lui ; il déchira ses vêtements, et il l'immola après lui avoir permis d'aller pleurer sur les montagnes le malheur de mourir vierge.» (O.C., t. 36, Oxford, 1994, p. 240-241.)

17. Voltaire suivait déjà cette démarche quand il parlait du baptême, dans la première des Lettres philosophiques.

18. Contes en vers et en prose, éd. citée, tome I, p. 242 et II, p. 197.

19. Formule par laquelle J. Fourastié définit l'humour, citée par J. Emelina, p. 130.

20. Malgré la longévité propre aux temps mythiques, en raison de laquelle Amaside conjure la vieille « par votre père, par votre mère, par votre nourrice, qui sans doute vivent encore » (364).

21. Faut-il rappeler que Loth a commis l'inceste avec ses deux filles? (Genèse XIX 30-38)

22. «A biblical 'conte philosophique' : Voltaire's Taureau blanc », XVIIIth Century French Studies, 1969, p. 55-69.

23. R. Pomeau, introduction citée, p. XLVII.

24. Dictionnaire de poétique et de rhétorique, Paris, P.U.F., p. 582.

25. L'ironie, Paris, 1964, p. 188.

\section{RÉSUMÉS}

Jugeant que l'intertextualité et l'humour impliquent l'un et l'autre une hétérogénéité discursive, nous avons choisi de les analyser dans un conte où Voltaire a emprunté à l'Ancien Testament son bestiaire et une partie de ses personnages pour suggérer, en jouant avec le merveilleux, que la Bible n'est qu'une fable parmi d'autres.

Mais à côté de l'intention satirique et polémique, de la désacralisation par le travestissement burlesque, il y a place pour le dérèglement ludique et la féérie. A côté du message explicite de l'énoncé se fait jour le charme discret de l'énonciation.

La fantaisie du conteur s'exerce sur des réminiscences bibliques en produisant de constants décalages qui, pour un lecteur complice, sont source d'humour: situations inattendues, comportements surprenants, dérapages chronologiques, pastiches insolites, paralogismes, disparates stylistiques, jeu entre le dit et le non dit.

Quand le conteur brode sur le canevas d'un imaginaire ancien des aventures cocasses, quand il tient avec impassibilité un discours incongru, en mimant le plus grand naturel malgré la présence d'incompatibilités arbitraires et de décrochages, il crée, par l'alliance de la virtuosité et 
de la feinte naïveté dans l'usage des allusions intertextuelles, un univers étrange, d'une poésie surréelle.

\section{AUTEUR}

\section{MARIE-HÉLĖNE COTONI}

Université de Nice-Sophia Antipolis 